\title{
Fractional-Order-Based System Identification for Heat Exchangers
}

\author{
Kin M. Li ${ }^{1}$, Mihir Sen ${ }^{2}$, Arturo Pacheco-Vega ${ }^{1}$ \\ ${ }^{1}$ California State University, Los Angeles \\ Los Angeles, CA 90032, USA \\ kli24@calstatela.edu; apacheco@calstatela.edu \\ ${ }^{2}$ University of Notre Dame \\ Notre Dame, IN 46556, USA \\ msen@nd.edu
}

\begin{abstract}
In this paper, we present a system identification (SI) procedure to build linear time-dependent fractional-order differential equation (FDE) models, that are able to describe time-dependent behavior of heat exchangers. The SI operation is carried out via global regression of an error-cost function by a simulated annealing optimization algorithm. The parameters in the models are: the order of the equation, the coefficients in it and - when necessary - the derivative of the initial condition. The Caputo definition of the fractional derivative, and the Mittag-Leffler function, are used to obtain the corresponding solutions. To test the SI approach, we consider a number of time-dependent analytical problems and experimental data from a shell-and-tube heat exchanger. In all cases, the resulting fractional-order model represents behavior of the system more accurately than typical integer-order models.
\end{abstract}

Keywords: Fractional calculus, System identification, Global regression, Mathematical model, Heat exchanger.

\section{Introduction}

Heat exchangers are important thermal systems with widespread applications, including: air conditioning, power generation, manufacturing, etc. The main goal of these devices is to maintain precise temperature conditions by controlling outlet temperatures of the working fluids in response to the operating conditions in a particular application. It is, thus, crucial to predict and control the behavior of these thermal devices. This calculation is difficult from a first-principles standpoint; geometry, turbulence, temperature dependent properties all add to the complexity of the problem. As a result, e.g., predictions are typically based on mathematical models derived from experimental data for specific heat exchangers. For steady state conditions, correlation equations in terms of Nusselt and Reynolds numbers are common [1]-[3]. However, modeling the dynamic behavior of a heat exchanger typically requires solving either partial differential equations (PDEs) or systems of ordinary differential equations (ODEs), which are computationally expensive and not suitable for real-time control purposes. Therefore, there is a need for compact and accurate mathematical models of time-dependent behavior of these systems.

Fractional-order equation models, which belong to the field of fractional calculus, have emerged as a possibility in system identification - the process of building mathematical models from data - of dynamic processes due to their potential to describe complex systems using a few terms. Fractional calculus has been known for sometime and has a history dating back to Leibniz [4]. In the last two decades the subject has broaden its applications in physics and engineering on a number of subjects, including: anomalous diffusion [5], robotic systems [6] and viscoelastic systems [7], among others. In thermal modeling, studies such as those of Aoki et al. [8], Pineda et al. [9], and Gabano and Poinot [10], demonstrate that fractional derivatives provide good approximations for describing the dynamic behavior of heat transfer processes. These results, along with those reported by Mayes [11] for heat-exchanger data, provide the rationale to develop fractional-order compact models that can accurately predict the dynamic behavior of heat exchangers.

This work focuses on a system identification (SI) technique based on global regression, that enables developing linear time-dependent fractional-order differential equation (FDE) models from heat exchanger experimental data. To this end, a brief background on fractional calculus is presented first. Then, we describe the system identification methodology, in which the error-cost function is minimized by the simulated annealing algorithm. To demonstrate its accuracy and effectiveness, the procedure is finally applied to a set of analytical problems, and to experimental data obtained from a 
shell-and-tube heat exchanger. In all cases, the fractional-order-based models are very accurate and, in the case of the analytical examples, perform better than their integer-order counterparts. The results can be extended to a number of different physical problems.

\section{Mathematical Background}

The history of non-integer calculus is almost as long as that of integer calculus itself. The first acknowledgment on the non-integer derivative can be traced back to a letter from L'Hopital to Leibniz inquiring about the existence of the half derivative, to which the reply stated that "it would be an apparent paradox, for which useful consequences will one day be drawn." Since then, the theory in non-integer calculus has gained significant progress, especially due to contributions from Laplace, Fourier, Abel, Louiville, and other 18th- and 19th-century mathematicians. However, it is only the last twenty years that non-integer calculus has acquired special importance in application areas. Such interest has been reflected by the number of publications devoted to the topic, including the classical books of Oldham and Spainer [12], Samko et al. [13] and, more recently, those of Podlubny [14] and Das [4], along with a number of review and scientific articles [15].

In essence, the field of fractional calculus generalizes the concept of a derivative beyond typical integer orders. It is concerned with the meaning of the "in-between" derivatives. For instance, the 3/2-derivative

$$
f^{(3 / 2)}(t)=\frac{d^{3 / 2} f}{d t^{3 / 2}}
$$

can be thought of as a derivative "in-between" the first and second derivatives. It is important to note that there is not a unique definition of a fractional derivative, but a number of them are possible as long as the definition itself satisfies a required set of mathematical rules [16].

Two of the most common definitions of the fractional derivative are those of Riemann-Louiville [13] and Caputo [17]. By starting with the Cauchy's formula for repeated integrals of the integer order $m$

$$
J^{m} f(t)=\frac{1}{(m-1) !} \int_{0}^{t}(t-\tau)^{m-1} f(\tau) d \tau ; \quad m \in \mathbb{Z}
$$

where $J^{m}$ is an integral operator, the Riemann-Louiville fractional integral of order $\alpha$, can be defined in terms of the Gamma function

$$
\Gamma(z)=\int_{0}^{\infty} e^{-t} t^{z-1} d t
$$

which allows replacing the term $(m-1)$ ! with $\Gamma(\alpha)$, as

$$
J^{\alpha} f(t)=\frac{1}{\Gamma(\alpha)} \int_{0}^{t}(t-\tau)^{\alpha-1} f(\tau) d \tau
$$

where $\alpha>0$ is an arbitrary positive real number, and $\tau$ is dummy variable of integration. Note that $J^{\alpha} f(t)=D^{-\alpha} f(t)$. Thus, for an integer $m$, such that $m-1<\alpha \leq m$, then

$$
D_{R L}^{\alpha} f(t)=D^{m} J^{m-\alpha} f(t)
$$

which defines the Riemann-Louiville fractional derivative, of order $\alpha$, as

$$
D_{R L}^{\alpha} f(t)=\frac{d^{m}}{d t^{m}}\left[\frac{1}{\Gamma(m-\alpha)} \int_{0}^{t} \frac{f(\tau)}{(t-\tau)^{\alpha+1-m}} d \tau\right]
$$


The Caputo definition of the fractional derivative of order $\alpha$, on the other hand, is given by

$$
D_{C}^{\alpha} f(t)=\frac{1}{\Gamma(\alpha-m)} \int_{0}^{t} \frac{f^{(m)}(\tau) d \tau}{(t-\tau)^{\alpha+1-m}}
$$

with $D_{C}^{\alpha} f(t)$ being defined as

$$
D_{C}^{\alpha} f(t)=D^{m-\alpha} J^{m} f(t)
$$

The subscripts $R L$ and $C$ in Eqs. (6) and (7) denote, respectively, Riemann-Louiville and Caputo fractional definitions. Regardless of the definition used, the Laplace transform is a powerful technique to solve equations involving fractional derivatives. For instance, the Laplace transform for the Riemann-Louiville definition is given by

$$
\mathcal{L}\left(D_{R L}^{\alpha} f(t)\right)=s^{\alpha} F(s)-\sum_{k=0}^{m-1} s^{k}\left(D^{\alpha-k-1} f(0)\right) ; \quad m-1<\alpha \leq m
$$

where it can be seen that the transformation of Eq. (9) to the Laplace domain, requires the following initial conditions: $D^{\alpha-k-1} f(0)$, for all $0 \leq k \leq m-1$. Since $\alpha$ does not need to be an integer number, it follows that the terms $D^{\alpha-k-1} f(0)$ are of fractional order. This poses a problem for applications in physical systems, where the initial conditions are typically given in terms of integer orders. On the other hand, the Laplace transform for the Caputo definition is given by

$$
\mathcal{L}\left(D_{C}^{\alpha} f(t)\right)=s^{\alpha} F(s)-\sum_{k=0}^{m-1} s^{\alpha-k-1} D^{k} f(0) ; \quad m-1<\alpha \leq m
$$

where, now the initial conditions necessary to evaluate Eq. (10) are prescribed as integer-order $D^{k} f(0)$, rather than fractional-order derivatives. Therefore, in the subsequent sections of the paper, we use the Caputo definition of a fractional-order derivative.

\subsection{System Identification Procedure}

In the context of fractional-order system identification (FOSI), the approach is similar to that based on integer-order equations, the main difference being the fractional-order nature of the mathematical model. Thus, as a first step, the model needs to be defined. For our purposes, we consider a linear fractional-order differential equation (linear FDE), defined as

$$
c_{1} \frac{d^{q} y(t)}{d t^{q}}+c_{2} y(t)=u(t)
$$

where $q$, with $n-1 \leq q \leq n(n \in \mathbb{Z})$, is the order of the model, $c_{1}$ and $c_{2}$ are constants, $t$ is the time-like independent variable, $y(t)$ is the output and $u(t)$ is an input to the system. In this paper $u(t)$ is a Heaviside function, since we are interested in operating conditions that take the device from one state to another; i.e., relaxation processes. The Laplace transform of Eq. (11), leads to

$$
s^{q} Y(s)-\sum_{k=0}^{n} s^{q-k-1} y^{(k)}(0)+\frac{c_{1}}{c_{2}} Y(s)=\frac{1}{c_{1} s}
$$

which can then be solved if the initial conditions for $y$, and its corresponding derivatives, are given. The inverse Laplace transform of Eq. (12) [4], generates the solution for $y(t)$, as

$$
y(t)=\sum_{k=1}^{n} B_{k} t^{k-1} E_{q, k}\left(-\frac{c_{2}}{c_{1}} t^{q}\right)+\frac{1}{c_{1}} t^{q} E_{q, q+1}\left(-\frac{c_{2}}{c_{1}} t^{q}\right)
$$


where $E_{q, k}$ is the two-parameter Mittag-Leffler function which, for integer orders, becomes a typical exponential function. The constants, $B_{k}$, represent the required initial conditions, as defined by

$$
B_{k}=y^{(k-1)}(0) ; \quad k=1,2, \ldots, n
$$

Importantly, the number of initial conditions is directly associated with the value $n$. If, for example, $q=1.75$, then $B_{1}=y(0)$ and $B_{2}=y^{\prime}(0)$ are required to compute the solution. Note that in Eq. (13), the first term corresponds to the solution of the homogeneous part of Eq. (11), while the second term represents the contribution from the Heaviside function. Once the model is set, the goal is then to find the values of the parameters in it; i.e., $q, c_{1}, c_{2}$ and, when the initial conditions are unknown, also $B_{1}$ and/or $B_{2}$, from the given data, which will provide the best-possible mathematical model of the system; i.e., the best-fit model.

To this end, we define an error-cost function $S_{u}$, to be the variance of the error between the model given by Eq. (11) and the data, as

$$
S_{u}=\frac{1}{N} \sum_{i=1}^{N}\left(y_{i}^{p}-y_{i}^{t}\right)^{2}
$$

where $N$ is the number of data points, $p$ refers to predictions from the model, and $t$ specifies the target data (either experimental or numerical). If we define the set of unknown parameters as

$$
P=\left\{q, c_{1}, c_{2}\right\}
$$

then,

$$
S_{u}(P)=\frac{1}{N} \sum_{i=0}^{N}\left(y_{i}^{p}(P)-y_{i}^{t}\right)^{2}
$$

and the problem reduces to finding the set $P$ that minimizes the objective function $S_{u}$.

The minimization process of $S_{u}$ (regression analysis), can be accomplished by either local or global optimization techniques, each of which have advantages and drawbacks [2]. In local regression, the algorithm seeks for the minimum value of the objective function nearest to a starting position. Typical local optimization algorithms (LOAs) are those based on the gradient, like the steepest decent or the Levenberg-Marquardt, among others. On the other hand, global optimization algorithms, of which genetic algorithms (GAs) and simulated annealing (SA) are examples, search in the entire domain to find the global minimum. However, computational costs of the latter are much higher than those of the earlier.

Following the investigations by Pacheco-Vega et al. [1, 18] in the framework of heat transfer correlations, and Li et al. [19] within the context of fractional-based models of complex systems, which demonstrate the non-uniqueness of the mathematical model obtained from regression analysis, the use of a global optimization technique is essential to find the optimum parameters in the model [Eq. (11)]. Therefore, in the following discussion the method of simulated annealing (SA), is used in to carry out the system identification.

A summary of the SA algorithm is given below, but additional details can be found in Aarts and Korst [20], among other literature. The steps are:

- Initialize the set of unknown parameters and temperature-like variable $T^{*}$.

- Perform random moves in the parameter space.

- The move is accepted according to the Metropolis Criterion: If new value is better, it is always accepted; otherwise, it is accepted with a probability of $p(\Delta f)=e^{-\frac{\Delta f}{T^{*}}}$.

- After each iteration, reduce temperature-like value $T^{*}$.

- Cycle until stopping criterion is satisfied. 


\section{Analytical Test Problems}

To demonstrate the robustness of the procedure, we apply the fractional system identification methodology to a number of linear and non-linear analytical problems that were proposed by Mayes [11], and compare the accuracy of the results from this work with those of Mayes [11], and with traditional integer models.

\subsection{Linear Problem}

The first problem considered is the solution of a linear third order differential equation, given by

$$
\frac{d^{3} y}{d t^{3}}+6 \frac{d^{2} y}{d t^{2}}+11 \frac{d y}{d t}+6 y=6 u(t)
$$

in the range $t \in[0,3 \cdot 5] . u(t)$ is a typical Heavyside function. The proposed linear fractional differential model is given in Eq. (11). For comparison purposes, a second-order differential equation

$$
c_{1} \frac{d^{2} y(t)}{d t^{2}}+c_{2} \frac{d y(t)}{d t}+c_{3} y(t)=u(t)
$$

along with the SI process outlined above for the parameters $\left\{c_{1}, c_{2}, c_{3}\right\}$, is also considered here. Note that, in Eq. (18), the initial conditions are set to $y(0)=y^{\prime}(0)=y^{\prime \prime}(0)=0$. Therefore, the constants in Eq. (13), $B_{1}$ and $B_{2}$ are both zero.

The corresponding results from the fractional-based model and the second-order equation, along with those of Mayes [11], are illustrated quantitatively and qualitatively in Table 1 and Fig. 1. The table shows that, not only the order of the model equations, and accompanying constants, are different among them, but also is the accuracy of their corresponding results. The closest approximations are provided by the fractional-based models, with values of $S_{u}$ within $6 \%$, and the worst are from the second-order equation, with more than a 30 -fold difference from the others.

Table 1: Model parameters for third-order linear problem [Eq. (18)].

\begin{tabular}{|c|c|c|c|c|c|}
\hline Model & $q$ & $c_{1}$ & $c_{2}$ & $c_{3}$ & $S_{u} \times 10^{4}$ \\
\hline Second order & 2 & 0.9929 & 1.8945 & 0.9037 & 31.9 \\
\hline This work & 1.722 & 2.2467 & 1.7362 & - & 1.21 \\
\hline Mayes [11] & 1.7130 & 2.2338 & 1.7359 & - & 1.28 \\
\hline
\end{tabular}

On the other hand, the approximation curves from the three models, shown along side the original data, are in agreement with the quantitative results of Table 1. From Fig. 1, it is apparent that the second-order model does not approximate the data very well, which is expected because the problem is of the third order, therefore more complex. However, it is surprising that the fractional model obtained here, and that of Mayes [11] using local regression (with very close values of the parameters), provide excellent approximations to the data. Both fractional models are very accurate in the region of $t \in[1,3]$, but their accuracy decrease at the extremes. At the tail end of the curve, it can be seen that the fractional models plateau at around $y=0.8$, whereas the data from Eq. (18) is still increasing. This is thought to be due to the lack of datapoints supporting the predictive capability of the model. Nevertheless, the outcome from the procedure provides a good rationale for using fractional-based differential equation models that are compact and accurate in describing systems that change from one state to another; i.e., relaxation processes. 


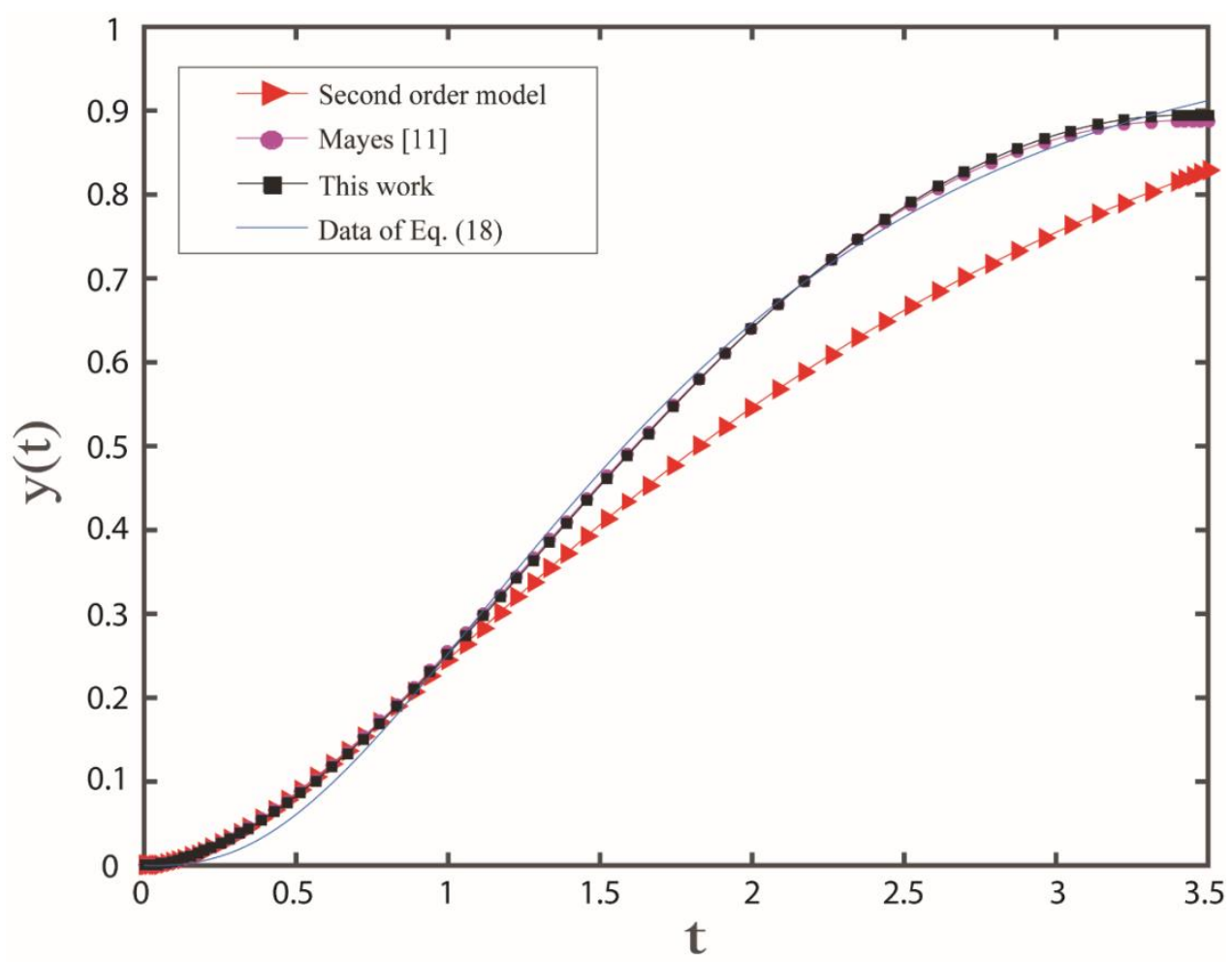

Fig. 1: Model approximations for third-order linear problem [Eq. (18)].

\subsection{Non-Linear Problem}

Next, we extend the analysis to a non-linear problem, and define the governing equation as

$$
\frac{d^{3} y}{d t^{3}}+6 \frac{d^{2} y}{d t^{2}}+11 \frac{d y}{d t}+6 y^{2}=6 u(t)
$$

where $u(t)$ is the Heavyside function. Note that this equation is similar to the linear problem considered before, the last term being modified with $y^{2}$. The time domain for this problem is, again, $t \in[0,3.5]$, and the initial conditions are $y(0)=y^{\prime}(0)=y^{\prime \prime}(0)=0$. As before, we apply the system identification procedure to find the parameters in both the fractional model given in Eq. (11), and the second-order equation (19). It is important to note that, this time, for the fractional-based model equation (11), the search is expanded to account for the value of $y^{\prime}(0)$; i.e., $B_{2}$ in Eq. (13), with $B_{1}=0$, that approximates the data the best. This is due to the fact that, in practical applications, initial conditions particularly that of the derivative - cannot be obtained in a straight-forward manner.

Results from this process are illustrated in Table 2 and Fig. 2. The table shows that, similarly to the previous example, the fractional model and that of Mayes [11] have different values of the parameters. This is likely due to the introduction of the parameter $B_{2}=y^{\prime}(0)$ in the search, along with the non-linearity of the problem and the fact that Mayes [11] used a local optimization algorithm, instead of the global regression used here. A comparison of the cost function, shows that the fractional models are substantially more accurate than that of the second-oder equation. Their corresponding values in $S_{u}$ show a tenfold improvement relative to that of the Mayes [11], and remarkably better than those of the integer-order model.

The aforementioned results are confirmed by the approximation curves shown in Fig. 2, where it is possible to observe that the fractional model developed here outperforms that of Mayes [11] in the entire range, most significantly in the two extremes, and is substantially better than the second-order solution. It can also be seen that this model also performs well at the tail end of the curve, as opposed to the linear test case, which can be attributed to the inclusion of parameter $B_{2}=y^{\prime}(0)$ in the search. 
Table 2: Model parameters for third-order non-linear problem [Eq. (20)].

\begin{tabular}{|c|c|c|c|c|c|c|}
\hline Model & $q$ & $c_{1}$ & $c_{2}$ & $c_{3}$ & $B_{2}$ & $S_{u} \times 10^{5}$ \\
\hline Second order & 2 & 0.8614 & 1.6737 & 0.813 & 0 & 570 \\
\hline This work & 1.589 & 1.3999 & 1.2185 & 0 & -0.2062 & 1.69 \\
\hline Mayes [11] & 1.777 & 2.0193 & 1.5662 & 0 & 0 & 13.9 \\
\hline
\end{tabular}

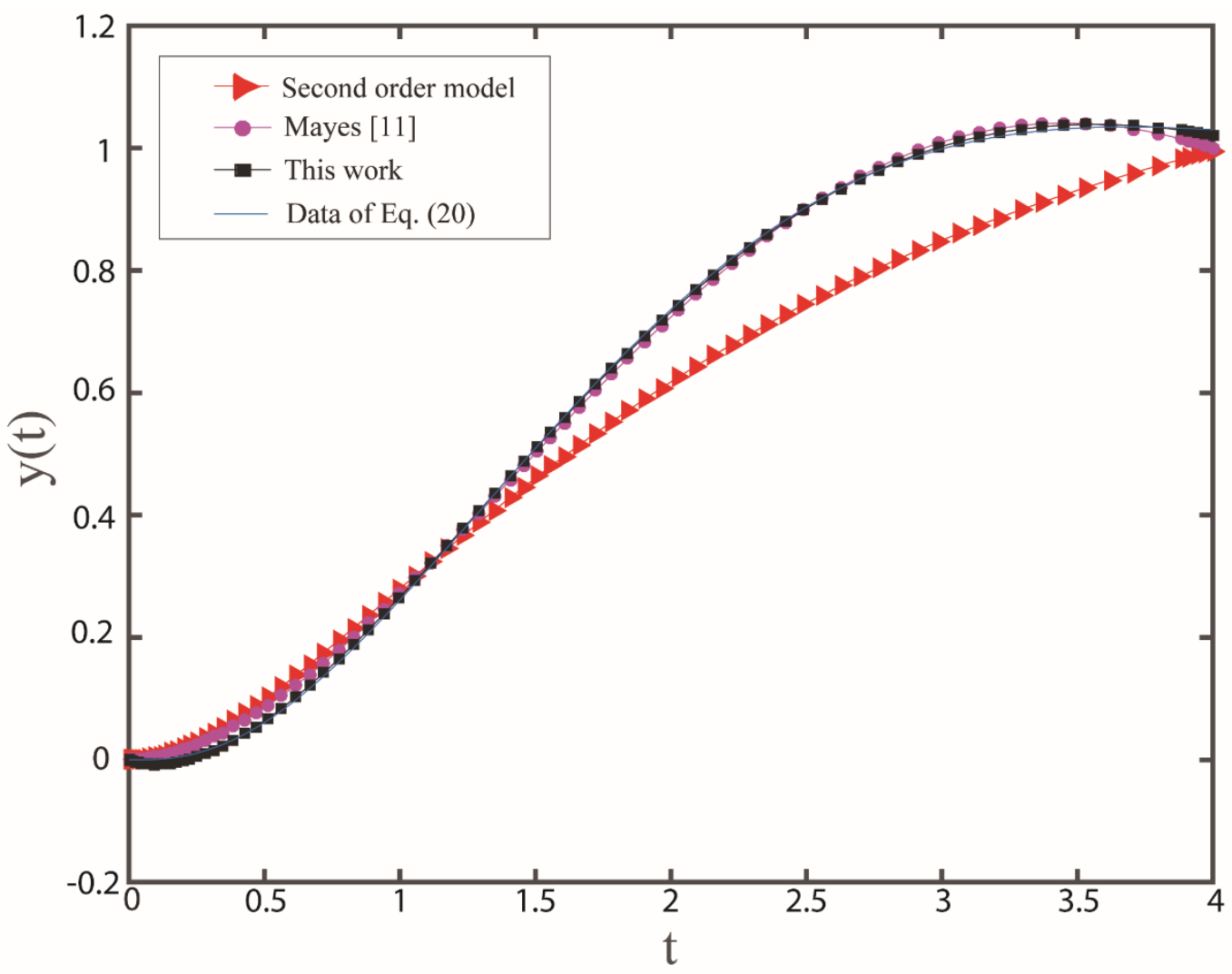

Fig. 2: Model approximations for third order non-linear problem [Eq. (20)].

\section{Shell-and-Tube Heat Exchanger Data}

In the previous section we have applied the fractional order system identification (FOSI) procedure to a set of analytical problems. In this section, the methodology is applied to experimental data from a shell-and-tube heat exchanger. Details about the experimental setup and the data have been reported in Mayes [11]; thus, here we only provide a brief description of the problem at hand.

The heat exchanger is illustrated schematically in Fig. 3. Shown in the figure are typical flow directions of the cold and hot fluid, along with the terminal inlet $T_{c}^{\text {in }}$ and $T_{h}^{\text {in }}$, and outlet $T_{c}^{\text {out }}$ and $T_{h}^{\text {out }}$, temperatures of both fluids. The tests were carried out under time-dependent conditions of the device, with the inlet temperatures and flow rates being held constant until thermal equilibrium was achieved. Subsequently, while maintaining the inlet temperature of both fluids and the hot-fluid mass flow rate at constant values, a step change in the flow rate of the cold fluid was then applied. Both inlet and outlet temperatures on the two fluids were recorded and stored in a PC for further analysis. 


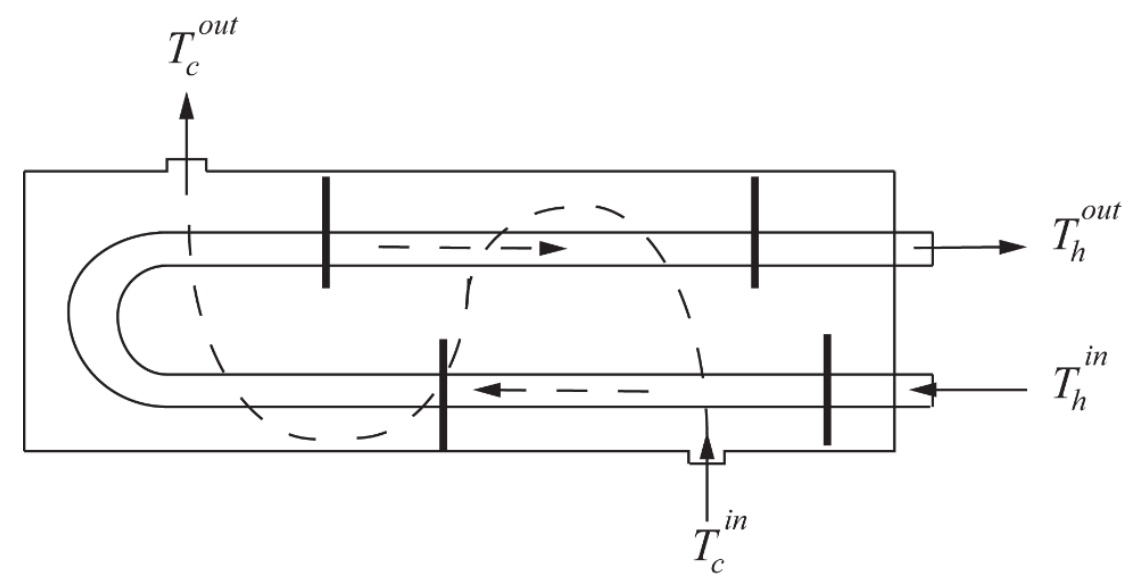

Fig. 3: Schematic of a typical shell-and-tube heat exchanger.

Since the objective of the study in [11] was to analyze the dynamic response of the hot-side outlet temperature due to a step change in the cold-fluid flow rate, then the focus is on the temporal behavior of the hot-fluid temperature $T_{h}^{\text {out }}$. Thus, we follow [11] and normalize the hot-fluid outlet temperature and the time variable. The dimensionless temperature $\theta$, and time $\tau$, are defined by

$$
\theta(t)=\frac{T_{h}^{\text {out }}(t)-T_{h}^{\text {out }}(0)}{T_{h}^{\text {out }}(\infty)-T_{h}^{\text {out }}(0)} ; \quad \tau=\frac{t}{\tau_{r}}
$$

where, $T_{h}^{\text {out }}(0)$ and $T_{h}^{\text {out }}(\infty)$ are, respectively, the initial value of the hot-fluid outlet temperature and its corresponding steady-state value; $\tau_{r}$ is the rise time, defined as the time required for $T_{h}^{\text {out }}(t)$ to reach $85 \%$ of $T_{h}^{\text {out }}(\infty)$. Now, $\theta(t) \in$ $[0,1]$. Again, the goal here is to find the parameters of a fractional model of the form (11) that fits the experimental data. For consistency, we define $\theta(t) \equiv y(t)$ and $\tau \equiv t$ so that Eq. (11) can be used, and apply the FOSI approach to the normalized data. For purposes of comparison, in addition to using the simulated annealing technique (SA), we also apply a local optimization algorithm (LOA) to find the best fit equation model (11). The parameters considered are: $\left\{q, c_{1}, c_{2}, B_{2}=\right.$ $\left.y^{\prime}(0)\right\}$, since $\theta^{\prime}(0) \equiv y^{\prime}(0)$ and the normalized initial value of the temperature is $\theta(0) \equiv y(0)=0$.

Comparison between the results from the fractional-based models obtained from SA and LOA are presented in Table 3 and Fig. 4. From the table, it can be seen that, the two fractional models have significantly different values of the parameters (particularly the order of the equation $q$ ) and the corresponding cost-function $S_{u}$. It is important to note that the accuracy of the model obtained from the SA is significantly higher than that of the model obtained by LOA; the value of $S_{u}$ of the latter being $33 \%$ higher than that of the earlier. This outcome is indicative of the non-uniqueness of the resulting fractional differential equation model, as discovered by Li et al. [19]. The qualitative results from the process, shown in Fig. 4, are in agreement with those presented in Table 3. From the figure it is possible to observe that, although both models behave similarly in the middle regions, i.e. $t \in[0.2,0.8]$, their predictions deviate at the two ends of the relaxation process, with the SA-based model being much closer to the original data. This implies that the system identification with a global regression algorithm, such as SA, provides the best possible fractional differential equation model.

Table 3: Model parameters for shell-and-tube heat exchanger data.

\begin{tabular}{|c|c|c|c|c|c|}
\hline Model & $q$ & $c_{1}$ & $c_{2}$ & $B_{2}$ & $S_{u} \times 10^{4}$ \\
\hline $\begin{array}{c}\text { Local } \\
\text { optimization }\end{array}$ & 0.9763 & -0.0356 & -0.0747 & 30.11 & 9.25 \\
\hline $\begin{array}{c}\text { Simulated } \\
\text { Annealing }\end{array}$ & 1.6708 & 0.3313 & 1.8067 & -0.1093 & 6.94 \\
\hline
\end{tabular}




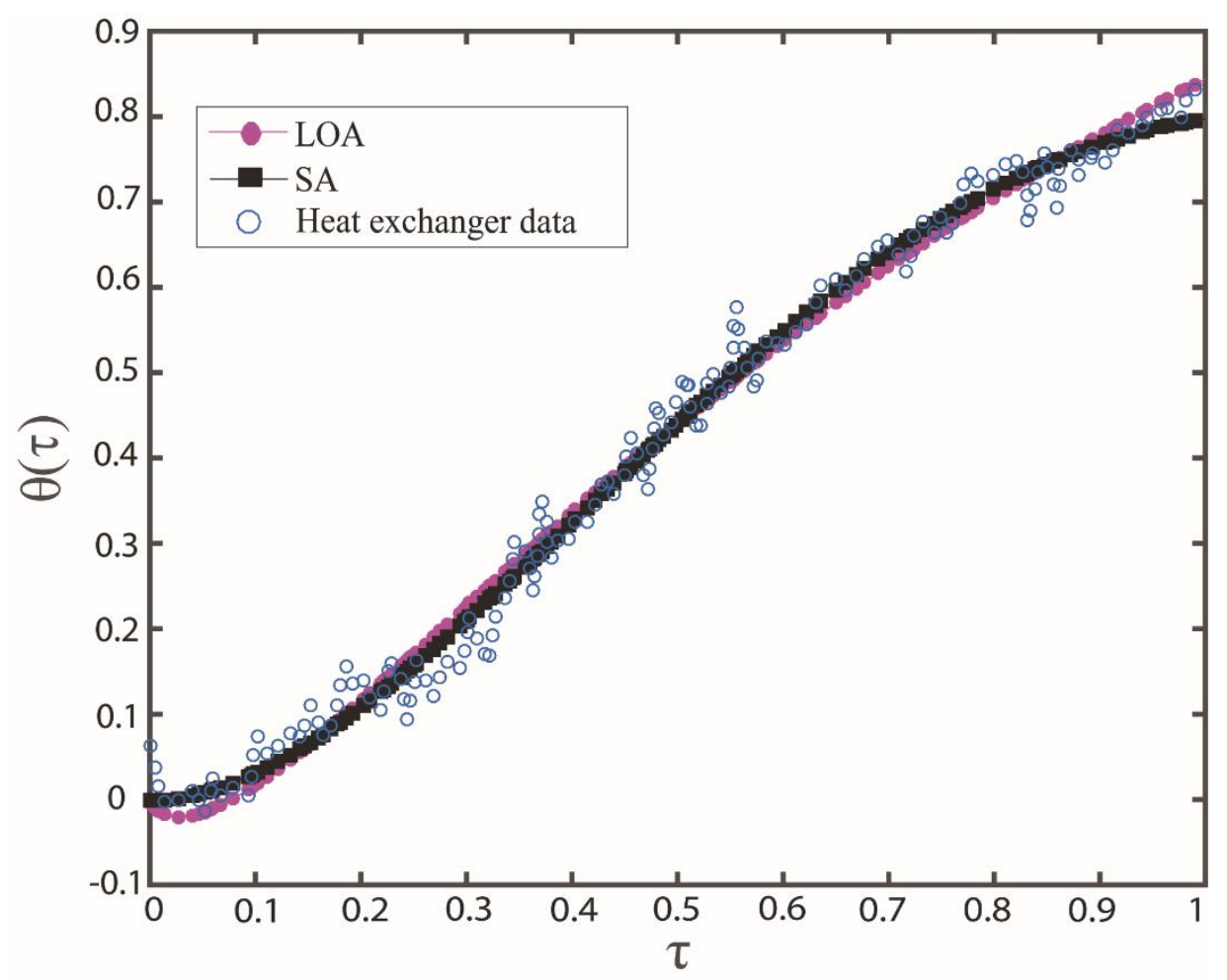

Fig. 4: Model approximations for shell-and-tube heat exchanger data.

\section{Conclusions}

Heat exchangers are important thermal systems with widespread applications, and for their control it is key to obtain an accurate description of their dynamic behavior. In this regard, fractional-order differential equations, derived via system identification, may provide compact and efficient models for prediction and control of these devices. In this work, a fractional-order-based system identification (SI) procedure has been proposed to build accurate models from data. The SI methodology is grounded on global regression, via the simulated annealing technique to provide the optimal model of the system. Its application to two analytical test problems, and to experiments from a shell-and-tube heat exchanger, has confirmed that the approach is accurate and robust. For both analytical problems, the fractional differential-equation models obtain here outperform their second-order counterparts. For the case of describing the dynamic behavior of the heat exchanger, the fractional-based model obtain via global regression provides better approximations to the data, than those obtained by SI with local optimization algorithms. Finally, results indicate that the initial conditions in the proposed models have significant impact in the accuracy of the approximation, especially at the extremes, and should be included in the search parameters. The methodology described here can be extended to model other physical systems.

\section{Acknowledgements}

Kin M. Li is the recipient of a CREST-CEaS fellowship, for which we are grateful. This work has been supported by an NSF HRD-1547723 grant.

\section{References}

[1] A. Pacheco-Vega, G. Diaz, M. Sen, K.T. Yang and R.L. McClain. "Heat rate predictions in humid air-water heat exchangers using correlations and neural networks," ASME J. Heat Transfer, vol. 123, no. 2, pp. 348-354, 2001.

[2] A. Pacheco-Vega, M. Sen and K.T. Yang, "Simultaneous determination of in- and over-tube heat transfer correlations in heat exchangers by global regression," Int. J. Heat and Mass Transfer, vol. 46, no. 6, pp. 1029-1040, 2003. 
[3] W. Cai, A. Pacheco-Vega, M. Sen and K.T. Yang, "Heat transfer correlations by symbolic regression," Int. J. Heat and Mass Transfer, vol. 49, no. 23-24, pp. 4352-4359, 2006.

[4] S. Das, Functional Fractional Calculus. Mumbai, India: Springer, 2011.

[5] L. Vlahos, H. Isliker, Y. Kominis and K. Hizanidis, "Normal and anomalous diffusion: A tutorial," ArXiv, (0805.0419v1), 2008.

[6] B. Goodwine, "Modeling a multi-robot system with fractional-order differential equations," in Proceedings of the IEEE International Conference on Robotics and Automation, Hong Kong, China, 2014, pp. 1763-1768.

[7] K. A. Lazopoulos, D. Karaoulanis and A. K. Lazopoulos, "On fractional modelling of viscoelastic mechanical systems," Mechanics Research Communications, vol. 78, pp. 1-5, 2016.

[8] Y. Aoki, M. Sen and S. Paolucci, "Approximation of transient temperatures in complex geometries using fractional derivatives," Heat Mass Transfer, vol. 44, pp. 771-777, 2008.

[9] S. M. Pineda, G. Diaz and C. F. M. Coimbra, "Approximation of transient 1D conduction in a finite domain using parametric fractional derivatives," ASME J. Heat Transfer, vol. 133, no. 7, p. 071301, 2011.

[10] J. D. Gabano and T. Poinot, "Fractional modelling and identification of thermal systems," Signal Processing, vol. 91, pp. 531-541, 2011.

[11] J. Mayes, "Reduction and Approximation in Large and Infinite Potential-Driven Flow Networks," Ph.D. dissertation, Dept. Mech, Eng., University of Notre Dame, Notre Dame, IN.

[12] K. B. Oldham and J. Spanier, The Fractional Calculus. New York, NY: Academic Press, 1974.

[13] S. G. Samko, A. A. Kilbas and O. I. Marichev, Fractional Integrals and Derivatives: Theory and Applications. Berlin, Germany: Gordon and Breach Science Publishers, 1993.

[14] I. Podlubny, Fractional Differential Equations. San Diego, CA: Academic Press, 1999.

[15] J. T. Machado, V. Kiryakova and F. Mainardi, "Recent history of fractional calculus," Commun. Nonlinear Sci. Num. Simulat., vol. 16, pp. 1140-1153, 2011.

[16] M. D. Ortigueira and J. A. Machado, "What is a fractional derivative?," Journal of Computational Physics, vol. 293, pp. 4-13, 2015.

[17] M. Caputo, "Linear models of dissipation whose $Q$ is almost frequency independent-II," Geophysical Journal International, vol. 13, pp. 529-539, 1967.

[18] A. Pacheco-Vega, M. Sen, K. T. Yang and R. L. McClain, "Genetic-algorithm-based-predictions of fin-tube heat exchanger performance," in Proceedings of the Eleventh International Heat Transfer Conference, Seoul, Korea, vol. 6, pp. 137-142, 1998.

[19] K. M. Li, M. Sen and A. Pacheco-Vega, "Fractional-derivative approximation of relaxation in complex systems," Submitted to: Complexity, 2018. (under review).

[20] E. H. L. Aarts and J. H. M. Korst, Simulated Annealing and Boltzman Machines. Tiptree Essex, Great Britain: Wiley, 1989. 